\title{
Rutas del poblamiento temprano en Chiapas, México. Una propuesta DESDE Los Sistemas de INFormación Geográfica (SIG)
}

\author{
Early Settlements in Chiapas, Mexico. A Proposal Using Geographic Information Systems (GIS) \\ Juan Ignacio Macías-Quintero \\ Stephanie Elizabeth Reyes-Ibelles
}

Resumen: Se ofrece una propuesta que emplea los Sistemas de Información Geográfica (SIG) para la búsqueda sistemática de sitios con ocupaciones precerámicas. A través de los SIG, se vislumbrará la posible ubicación y uso de rutas de menor costo usadas de forma recurrente por grupos de cazadores recolectores durante el poblamiento del sureste mexicano (Chiapas), de forma específica en el área de la Depresión Central a finales del Pleistoceno (10000-8000 a.p.). La escala de análisis tratada posee más detalle y precisión que la de otros estudios. Se espera evaluar en el futuro los alcances y la efectividad de los SIG para predecir la ubicación de sitios relacionados con el poblamiento temprano en América.

Palabras clave: sistemas de información geográfica, arqueología del paisaje, poblamiento temprano, precerámico, cazadores recolectores, pleistoceno.

Abstract: We discuss a proposal to use geographic information systems (GIS) to undertake a thorough search of archaeological sites with preceramic settlements. We use GIS to discern the location and routes used by hunter-gatherer groups who colonized southeastern Mexico (Central Depression of Chiapas) during the late Pleistocene (ca. 10,000-8,000 BP). Our analytical scale contains a higher degree of detail and accuracy than previous studies. We hope to evaluate the scope and effectiveness of GIS for studies of early settlements in the Americas.

Keywords: geographic information systems, landscape archaeology, early settlements, preceramic, Pleistocene, hunter-gatherers.

Juan Ignacio Macías Quintero, doctor en Antropología por el Instituto de Investigaciones Antropológicas de la Universidad Nacional Autónoma de México. Profesor-investigador en la Facultad de Humanidades de la Universidad de Ciencias y Artes de Chiapas, México. Temas de especialización: arqueología de paisaje, arte rupestre, sociedades mesoamericanas, cazadores recolectores. Correo electrónico: juan.macias@unicach.mx.
Stephanie Elizabeth Reyes Ibelles, pasante en Arqueología en la Universidad de Ciencias y Artes de Chiapas, México. Temas de especialización: poblamiento temprano, cazadores recolectores, osteología. Correo electrónico: stephanie.reyes.ibelles@gmail.com.

Enviado a dictamen: 19 de septiembre de 2017

Aprobación: 16 de abril de 2018

Revisiones: 1 
U no de los temas más controvertidos en los estudios arqueológicos se refiere al poblamiento temprano del continente americano. En estos debates se discuten las posibles rutas y momentos en los que los primeros humanos llegaron a América, y si esta colonización y dispersión poblacional se trató de un solo proceso proveniente del norte y dirigido hacia el sur, pasando de Asia a Alaska por el estrecho de Bering, o si los desplazamientos se produjeron de sur a norte, desde Sudamérica a otros puntos del continente americano. Asimismo, otras discusiones se han enfocado en considerar que el "poblamiento temprano" en realidad se trató de varios poblamientos, con diversos orígenes, rutas y momentos, de manera que se formaron tradiciones tecnológicas paralelas y con respuestas variadas a los paleoambientes circundantes. ${ }^{1}$

El territorio que actualmente ocupa Chiapas es un enclave idóneo para examinar con mayor detalle estos procesos, a partir de la reconstrucción de las rutas por las cuales el tránsito de poblaciones humanas tempranas pudo ser factible. Un ejemplo notable de la modelación de rutas de tránsito de los primeros pobladores del continente americano fue desarrollada por Anderson y Gillman (2000), quienes por medio de los Sistemas de Información Geográfica (SIG) describieron las posibilidades de poblamiento considerando condiciones de terreno, clima y localización de sitios pleistocénicos (ver Mapa l).

Los resultados de sus trabajos mostraron la ubicación de rutas óptimas para el flujo constante de personas en el paisaje y, dentro del modelo que propusieron, el territorio del actual estado de Chiapas funge como un puente natural que une el altiplano mexicano con Centroamérica a través del Istmo de Tehuantepec y la costa del Soconusco. Sin embargo, dada la escala de territorio usada en su estudio - prácticamente desde Alaska hasta la Patagonia-, así como la ausencia de información de sitios pleistocénicos en Chiapas, surge la falsa impresión de que al interior del territorio no fue factible una colonización temprana.

Posteriormente, Acosta y colaboradores (2016) elaboraron una propuesta más detallada para la reconstrucción de las rutas que conectarían sitios paleoindios de Norteamérica con Centroamérica. Para ello, utilizaron una mejor base de datos y un Modelo de Elevación Digital (DEM por sus siglas en inglés) con mayor resolución que facilitó crear distinciones sobre las circunstancias de poblamiento de acuerdo con la variabilidad de tradiciones líticas y las posibilidades que ofrecía el terreno. Dichas posibilidades se reflejaron por medio de rutas de menor costo para el tránsito de personas a lo largo del territorio que actualmente ocupan el sur de Estados Unidos, México y Centroamérica. Al ser la escala territorial menor que la utilizada por Anderson y Gillam (2000), sus resultados fueron más puntuales en la reconstrucción de los movimientos de grupos paleoindios durante sus procesos de migración y colonización.

No obstante, aun con los anteriores aportes, nos enfrentamos a la problemática de saber si tales reconstrucciones pueden ayudar a la detección de áreas con mayor potencial para albergar sitios con ocupaciones precerámicas, así como de los territorios usualmente transitados por grupos paleoindios en sus rutas de migración y explotación del paisaje. Un estudio que pudiera arrojar información sobre ese tópico sería de mayor utilidad no sólo para la documentación y registro de los nuevos sitios, sino para arrojar luz sobre los procesos de poblamiento temprano en las Américas.

En la presente investigación se retomará la información disponible sobre sitios tempranos en Chiapas y, a través de la recreación de las condiciones del terreno por medio de DEM en un área específica - la Depresión Central de Chiapas - , se mostrarán las posibles vías optimas de tránsito entre estos sitios con el fin de recrear, predecir y reconstruir algunas de las rutas posibles de poblamiento empleadas por los cazadores recolectores a finales del Pleistoceno (ca. 10000 a.p.).

El interés se centra en el diseño y aplicación de métodos que permitan la búsqueda y detección de sitios tempranos de una manera más sistemática y precisa, al situar puntos en el paisaje con alto potencial 
para la exploración arqueológica detallada y, con ello, contribuir a los debates que buscan caracterizar el proceso del poblamiento temprano en América. El presente análisis es sólo una primera aproximación a una problemática añeja; dado que la investigación todavía se encuentra en desarrollo, los resultados aquí expuestos son provisionales.

\section{Rutas y modelos de colonización temprana en América. El corredor libre de hielo}

Algunos estudiosos han señalado que existió un puente terrestre que unía Asia y América durante diversos periodos glaciares, dado que la profundidad del mar en esa región es escasa. El puente terrestre hoy conocido como Beringia se trata de una amplia región que en su momento contó con los recursos para atraer fauna terrestre y poblaciones humanas (Dixon, 1999).

Para los partidarios del modelo Clovis First, el poblamiento americano debió llevarse a cabo durante el final de la última gran glaciación denominada de Wisconsin (ca. 25000 a 11000 años a.p.). En este modelo existen dos versiones: la primera propone que una migración procedente de Asia sólo pudo realizarse cuando los dos grandes bloques glaciares que cubrían Norteamérica estuvieron derretidos, entre 11000 y 12000 a.p. (Dixon, 1999; Lanata, 2011). La segunda versión sostiene que el poblamiento pudo realizarse antes de la fecha arriba expuesta, cuando se habría abierto entre los dos glaciares un hipotético "corredor libre de hielo", el cual consistiría en una delgada franja de tierra expuesta que permitiría el tránsito a través del área que ahora ocupa Alberta, en Canadá (Dixon, 1999; Fiedel, 1996). Pero en diversos estudios geológicos y paleoecológicos (Jackson et al., 1996) se ha subrayado que este supuesto corredor existió solamente a finales del periodo de Wisconsin, cuando el continente ya estaba poblado; además, las condiciones climáticas y ambientales predominantes en el corredor lo hacían prácticamente inviable para el poblamiento humano, incluso para otros mamíferos pleistocénicos.

\section{La ruta del Atlántico Norte}

En este modelo, se postula que el poblamiento americano se pudo dar por la costa del Atlántico Norte, procedente de Europa. En este proceso, la cultura solutrense del paleolítico superior europeo que entre los años 25000 y 18000 a.p. estuvo presente en el norte de España y Francia, habría arribado a América mediante el empleo de embarcaciones hechas de piel que podrían haber permitido la travesía por el Atlántico, considerando que durante el último máximo glacial las costas expuestas de Europa y América estarían más cercanas que en la actualidad - aproximadamente 2250 kilómetros-y permitirían llegar a la costa de Norteamérica (Stanford y Bradley, 2002; Bradley y Stanford, 2004).

Sin embargo, muchas de las características mencionadas como comunes entre Clovis y la cultura solutrense no lo son tanto. Por ejemplo, las sociedades solutrenses emplearon profusamente arte rupestre en sus depósitos, además de una gran cantidad de instrumentos en hueso, mientras que los Clovis no; sin mencionar, las enormes distancias que existen no sólo geográficamente, sino también en el tiempo, pues al menos 5000 años de distancia separan a ambas culturas (Acosta, 2008).

\section{La ruta por la costa del Pacífico}

Otros investigadores, en la línea del poblamiento proveniente de Beringia, sugieren como ruta las zonas costeras del Pacífico noroeste. Fladmark $(1979,1986)$ planteó la posibilidad de que los primeros americanos hubieran colonizado gradualmente refugios costeros libres de hielo a lo largo de la costa de Beringia y del noroeste americano mediante embarcaciones. Esto parece ser corroborado por estudios geológicos y paleoecológicos, que indican que las costas de Beringia pudieron ser ecosistemas potencialmente más ricos en recursos que las tierras interiores, lo que sugiere la existencia de un corredor migratorio habitable y navegable a lo largo del macizo continental (Josenhans 
et al., 1997). De esta manera, se explicaría por qué los sitios de la costa sudamericana tendrían fechamientos tan tempranos, incluso anteriores a Clovis, a diferencia de otros sitios del interior del continente (Acosta, 2008).

\section{Las culturas precerámicas en Chiapas ${ }^{2}$}

Chiapas es una entidad federativa localizada en el sureste de México que colinda al este con la República de Guatemala y al sur con el océano Pacífico (ver Mapa 2). El territorio se ha convertido en un punto importante para el estudio del poblamiento temprano ya que cuenta con notables evidencias que indican la presencia de los primeros pobladores. ${ }^{3}$

A finales del periodo de Wisconsin (25000 a 11000 años a.p.) la región central de Canadá y Estados Unidos, la meseta central del Altiplano Norte y la cuenca del Valle de México se caracterizaron por un patrón climático más frío y húmedo, que a su vez propició la existencia de extensos pastizales (Johnson et al., 2006). Por su parte, la información paleoambiental recuperada en la cueva de Santa Marta, en el actual municipio de Ocozocoautla, indica un patrón climático más fresco y seco que en la actualidad, con vegetación predominante de tipo sabana y bosques mesófilos, perennifolios y caducifolios alternantes (Acosta, 2010 y 2014). Asimismo, análisis realizados por isótopos estables de carbono y oxígeno a dientes fósiles indican condiciones secas similares a las de una sabana en la Depresión Central (Pérez-Crespo et al., 2015).

La presencia de sociedades cazadoras en el territorio chiapaneco se ha podido documentar mejor gracias a las exploraciones efectuadas en la región del Soconusco, en la costa del Pacífico. Se trata de pequeños grupos dedicados a la explotación de antiguos ecosistemas costeros, identificados a través de los montículos denominados "concheros" (Voorhies et al., 2002). Los pobladores de esos montículos, conocidos como Chantuto, ocuparon la región costera desde el periodo Arcaico Medio (7000/6000-5000 a.C.).

Otros reportes derivan de los hallazgos realizados en las tierras altas de Chiapas, en específico en los valles de San Cristóbal, Aguacatenango y Teopisca. Estas áreas son relevantes, en virtud de la polémica levantada a raíz de los hallazgos que indican una secuencia de ocupación que posiblemente data desde finales de Pleistoceno hasta el año 3500 a.p. (García y Santamaría, 1982; Pincemin, 1990). Los citados valles se ubican en altitudes promedio entre 1800 y 1700 metros sobre el nivel del mar. Ambos conforman cuencas cerradas, y en algún momento contuvieron lagunas, una de ellas actualmente seca - Teopisca- En esta última se localizaron nueve sitios que muestran una posible ocupación temprana o arcaica. En Aguacatenango ocho sitios fueron registrados sobre las terrazas superiores circundantes a las lagunas, en donde se reportaron artefactos como raspadores, buriles, perforadores y muy pocas puntas de proyectil diagnósticas, las cuales, de acuerdo con García Bárcena (1982) son similares a las de tipo Lerma, Abasolo y Gary ubicadas en un rango cronológico muy impreciso (ca. 7000 a 2000 a.p.). Las materias primas comúnmente usadas para elaborar los artefactos eran jaspe, pedernal y cuarzos de la región (García, 1982; Pincemin, 1990).

En el valle de Teopisca, Lorenzo reportó material lítico que era manufacturado para actividades de corte por desgaste. Se trata de herramientas obtenidas por percusión directa que Lorenzo las asoció a otros sitios pleistocénicos ubicados en Nicaragua, con una dudosa antigüedad que se remonta hasta los 20000 años a.p. Aunque los artefactos no mostraron una asociación clara con estratos fechables, se le denominó a esta etapa "Horizonte de pre-puntas de Proyectil" (Lorenzo, 1977).

Adicionalmente, se identificó en el valle de San Cristóbal de Las Casas el sitio llamado Fogótico, con sedimentos fechados en 8600 y 6400 a.p., aunque en los reportes no se indica cuáles de los artefactos encontrados pertenecen a dicho periodo. En ese mismo valle destacan también los hallazgos de puntas de proyectil descritos por Lee (1985), en donde hoy se ubica el edificio Telmex en el centro de la ciudad de San Cristóbal de Las Casas. Aunque Lee no proporciona detalles sobre el contexto ni fechamientos absolutos de los artefactos, en su reporte muestra: una punta bifacial lanceolada con una leve concavidad basal y retoque paralelo convergente -ipreforma de Clovis? -, puntas con pedúnculo 
prominente y rectangular, posiblemente tipo "Gary", además de una punta de proyectil de forma triangular, con biselado alterno-opuesto, pedúnculo ancho y recto con aletas basales divergentes, muy similares a las puntas "Pedernales" reportadas por Flannery (1986) en Oaxaca, fechadas con una antigüedad de $8860 \pm 180$ a.p. Sin embargo, esa última punta también guarda similitud con las puntas "Xaagá" descritas por Winter (2014) en Oaxaca. Este autor estima que tales puntas se ubicarían cronológicamente en la fase "jícaras", es decir, entre 5360 y 6200 años a.C. Por otra parte, en Belice esas puntas suelen ser descritas como "Lowe", con un rango temporal estimado entre 2500-1900 a.C. o quizá más antiguo (Stemp et al., 2016).

La importancia de los hallazgos anteriormente descritos y el potencial que tienen los valles y cuencas endorreicas situadas en la región de Los Altos de Chiapas es innegable, sin embargo, las fechas de los artefactos "diagnósticos" no están del todo libres de debate y corroboración. De igual modo, muchos de los datos recuperados en las excavaciones de GarcíaBárcena aún no han sido analizados con detalle (Santamaría, 2014), de ahí que se torna indispensable subrayar que la presencia de poblaciones pleistocénicas en Los Altos de Chiapas es hipotética.

La presencia humana temprana en Chiapas ha sido mejor evidenciada en las cuevas de Santa Marta y Los Grifos en Ocozocoautla. Santa Marta es sin duda uno de los sitios con ocupación humana temprana más antiguos y mejor documentados de México y del trópico americano. ${ }^{4}$ Las cuevas se ubican en un ecosistema distinto al de Los Altos de Chiapas, en una meseta situada en Ocozocoautla con una altitud promedio entre los 800 y los 900 metros sobre el nivel del mar, en el extremo occidental de la Depresión Central.

El caso de la cueva de Santa Marta es todavía más trascendental en los estudios del poblamiento temprano. Investigaciones recientes emprendidas por Acosta $(2008,2011$ y 2014) han permitido demostrar ocupaciones más antiguas que las detectadas en sus trabajos por García y MacNeish (con fechas de 10 460 550 a.p). Lo más intrigante de los descubrimientos de Acosta y su equipo fue que las primeras ocupaciones no poseían características de los grupos paleoindios de Norteamérica, por ejemplo puntas de proyectil acanaladas tipo "Clovis" diseñadas para la cacería de megafauna. En lugar de esta tecnología especializada, los habitantes de Santa Marta elaboraban herramientas con un carácter más expeditivo y diverso (Acosta, 2008 y 2014). Es decir, utilizaban lascas poco especializadas, la talla bifacial no derivaba en artefactos diagnósticos, y también aprovechaban otras materias primas como el hueso, concha y madera; además de ello, explotaban un rango más amplio de nichos ecológicos sin la necesidad de elaborar herramientas muy sofisticadas (Acosta, Gómez y García, 2016).

Por otro lado, la cueva de Los Grifos ubicada a trescientos metros de Santa Marta — con fechas de 9540 \pm 150 a.p.- posee la peculiaridad de haberse encontrado en ella dos puntas de proyectil diagnósticas de los primeros pobladores americanos tanto de Sudamérica como de Norteamérica, es decir, puntas acanaladas tipo "Clovis" y puntas con pedúnculo de base ampliada conocidas también como "Colas de Pescado" (Acosta, 2008 y 2014; Dillehay et al., 1992). Los hallazgos sugieren que grupos con estas tecnologías arribaron al menos mil años después que los cazadores de tecnología expeditiva detectados en Santa Marta. La información proveniente de la cueva de Los Grifos también es significativa para el desarrollo de modelos que discutan procesos de migración y transmisión tecnológica recurrente en las poblaciones paleoindias, en virtud de ser un lugar de convergencia de tradiciones tecnológicas usualmente distanciadas en el espacio.

Los casos ya referidos resaltan la importancia de Chiapas y de las áreas tropicales para la comprensión de los procesos de migración y poblamiento temprano en el Nuevo Mundo. A pesar de que algunos de los asentamientos son controversiales por la escasa confiabilidad de sus fechas, las áreas estudiadas por García-Bárcena y colaboradores aún pueden ser revisadas y sus propuestas evaluadas. Los casos mejor documentados en las tierras bajas de Chiapas indican la presencia de cazadores recolectores con estrategias variadas de adaptación y uso del entorno. De acuerdo con Acosta (2014), algunos de estos grupos — cueva de 
Santa Marta - ya aprovechaban variedades de maíz $(Z e a)$, quizá algún teocintle alóctono, cacao (Theobroma sp.), tomate (Physalis sp), nanche (Brysonima crassigola) e higo (Ficus cooki). Lo ya señalado, a su vez, sugiere que la variedad de pobladores tempranos con orígenes y caracteres diversos es mayor de lo que comúnmente se pensaba.

\section{Procedimientos de análisis}

En cualquier paisaje dado, una multitud de factores medioambientales como la ubicación de agua potable, tierra arable, recursos maderables y materias primas estratégicas pueden influir en la elección del lugar para asentarse (Jones, 2006). A ello se le pueden sumar también componentes de carácter ideológico, político y social; en este sentido, conocer la totalidad de factores influyentes puede ser muy difícil, dado que no se suele tener conocimiento del sistema social completo (Jones, 2006).

La integración de distintos enfoques en la interpretación de paisajes arqueológicos es posible a través de los SIG porque este sistema ofrece la posibilidad de visualizar y analizar múltiples capas de datos, tanto colectados como modelados de forma hipotética (Chapman, 2006). Asimismo, permiten establecer procedimientos para que la realidad pueda ser modelada y susceptible de estudio; un modelo sería, entonces, una representación simplificada de la propia realidad. ${ }^{5}$

Esencialmente, un SIG es una "base de datos espaciales", es decir, un conjunto de datos que pueden ser fácilmente almacenados, gestionados y analizados en términos de su posición en el mundo, aunque en un sentido virtual. Los SIG sólo simulan el mundo físico y, en el caso de la arqueología, pueden demostrar cómo a veces esta simulación podría ser la forma más certera de acercarse a las realidades que se estudian del pasado (Chapman, 2006).

La simulación se puede entender como un recurso metodológico empleado ante la imposibilidad de operar directamente sobre el sistema real (Fábrega, 2004). De acuerdo con este planteamiento, el comportamiento de un fenómeno se replicaría de forma virtual para entender con mayor facilidad la naturaleza de la interacción entre las partes que lo conforman (Wheatley y Gillings, 2002; Fábrega, 2004).

\section{Creación de escenarios y modelos predictivos}

Las ventajas prácticas de los modelos predictivos se derivan del hecho de que pueden ser aplicados a zonas que aún no han sido prospectadas, es decir, donde no se conoce la ubicación de los sitios (Warren y Asch, 2000; Trujillo, 2011). Al promover esquemas bajo lógicas específicas - por ejemplo, la cercanía de recursos estratégicos o la accesibilidad del terreno para transitar-, los arqueólogos pueden dirigir sus prospecciones de forma más puntual para, así, optimizar los procesos de investigación en campo. También los modelos predictivos son de utilidad para producir esquemas de manejo y preservación de recursos arqueológicos de forma sistemática y eficiente. Consecuentemente, el éxito de un modelo predictivo sólo puede ser corroborado en virtud de las exploraciones en campo que se lleven a cabo.

\section{Procesamiento de la información}

Para realizar la reconstrucción de las rutas de movimiento de los grupos de cazadores recolectores en el territorio de Chiapas, fueron empleadas distintas fuentes de información, entre las que se cuentan:

a. Información georreferenciada de sitios arqueológicos con reporte de ocupaciones tempranas o abrigos rocosos. ${ }^{6}$

b. Información georreferenciada de yacimientos de fósiles pleistocénicos en los que, si bien no se reporta presencia humana, son puntos con potencial para exploraciones posteriores. ${ }^{7}$

c. Información cartográfica digital (vectorial) del estado de Chiapas a escala 1:50000 del INEGI.

La zona delimitada para la creación del DEM comprende las cuencas del sistema del Grijalva y las 
subcuencas alimentadoras de la Depresión Central de Chiapas, y abarca una extensión de 3924823 metros cuadrados. Pudo obtenerse un DEM con una resolución por pixel de veinticinco por veinticinco metros para obtener mayor precisión en la representación del terreno (ver Mapa 3). El proceso no fue sencillo, ya que nos topamos con la sorpresa de que existen zonas de la porción oriental de la Depresión Central de Chiapas que carecen de información vectorial. Además, la información cartográfica - vectores - del mosaico de cartas topográficas a escala 1:50000 del INEGI no empataban entre sí. Por lo tanto, recurrimos a elaborar cotas con equidistancias de veinte metros por medio del software Global Mapper ${ }^{\mathrm{TM}}$. La fotogrametría generada de la imagen satelital de Google Earth ${ }^{\mathrm{TM}}$ proporcionó las altitudes necesarias para la generación del DEM de toda el área correspondiente a la Depresión Central de Chiapas (ver Figura 1).

Los mapas que representan el costo de superficie se basan en el análisis de la constante relación que existe entre el incremento de la inclinación de una pendiente y el incremento del esfuerzo proporcional conforme dicha pendiente se hace más pronunciada, es decir, cuanto más inclinada sea la superficie, mayor costo requiere caminar sobre ella (ver Mapas 4, 5 y 6).

El modelo de la pendiente define un ráster basado en un DEM, donde cada una de las celdas contiene distintos valores de altitud que pueden expresarse en porcentaje o grados (Chapman, 2006). Posteriormente, la información recibió un nuevo tratamiento y se efectuó un mapa que indicara la dirección en que las pendientes se distribuían. El siguiente paso consistió en generar un mapa que calculara una relación de costo/distancia, es decir, que indicara cómo se incrementaba el costo acumulado para cada pixel a partir de un punto específico en el paisaje.

El último paso consistió en que el programa proyectara la "ruta óptima" entre sitios; la herramienta de ruta óptima permite trazar un vector que simula el tránsito de menor costo entre dos puntos de acuerdo con la fricción que generaría el movimiento en la superficie. Las derivaciones de los anteriores ejercicios se exponen en el Mapa 7 (ver Mapa 7).

\section{Discusión}

El resultado de los análisis anteriormente descritos muestra un mapa con el relieve detallado de la Depresión Central de Chiapas que sugiere las rutas de tránsito más viables por las que debieron de circular los habitantes de Santa Marta al visitar otros nichos ecológicos. Se sugieren las rutas más viables, lo cual no significa que en algún momento de la historia un grupo de cazadores que migrase de un punto a otro del territorio no optara por trasladarse por cualquier otra ruta. Con la expresión "rutas más viables" se hace referencia a los trayectos transitados con mayor frecuencia y que, por lo tanto, tendrían más posibilidad de albergar trazos materiales como producto de actividades reiteradas a lo largo de cientos o miles de años - por ejemplo, áreas de caza, campamentos efímeros o áreas de destazamiento, abrigo, recolección o extracción de materias primas-

La cueva de Santa Marta, además de ser uno de los sitios precerámicos mejor documentados en Chiapas (Acosta, 2008), es también calificado como un "campamento base". En los espacios de este tipo, la permanencia de una familia o de un grupo de familias solía ser prolongada, y son los lugares que guardan evidencia de una mayor cantidad de actividades domésticas tales como descanso, preparación de alimentos, almacenamiento, deshecho y trabajo. Del mismo modo, eran los puntos desde donde se efectuaba excursiones más específicas con el fin de recolectar o cazar.

Las líneas o vectores que conectan Santa Marta con otros sitios de Los Altos y con los yacimientos de fósiles de la Depresión Central marcarían el área y los alrededores con mayor potencial para albergar restos de acciones de los grupos de cazadores itinerantes. Estos argumentos y expectativas se basan en la noción de que la movilidad en el paisaje por parte de los cazadores recolectores persigue una lógica fundamentada en una relación de costo/beneficio (Bettinger, 1991; Winterhalder, 2003), lo cual significa que la razón para moverse por esas rutas y no por otras 
estaría dada por la disponibilidad y la facilidad para cazar y recolectar recursos cuyo beneficio fuera mayor al costo relacionado con la distancia hacia esos nichos desde un punto determinado (Macías, 2017).

Al considerar esas variables en el programa, las rutas reflejadas en el mapa revelaron no sólo los lugares por donde era más fácil trasladarse, sino también dónde se localizarían idealmente las zonas con recursos notables para congregar a los cazadores del pleistoceno. Igualmente, los movimientos y la explotación de nichos específicos — riberas de río, mesetas, montañas - se relacionaría con las posibilidades ecológicas que ofreciera cada zona de acuerdo con los cambios de estación anual (Butzer, 1989). Empero, los estudios paleoambientales necesarios para reconstruir la variedad y cantidad de recursos disponibles en las etapas finales del pleistoceno aún son escasos; su eventual documentación será de suma importancia para la mejora del modelo aquí discutido.

Es interesante observar que, si el territorio de los cazadores de Santa Marta fuera tan amplio como para visitar los puntos aquí señalados, tendrían la posibilidad de explotar recursos y ecosistemas variados - de la meseta de Ocuilapa a las llanuras bajas de la Depresión Central y las zonas montañosas de Los Altos-. Tal como fue puntualizado inicialmente, en esta última zona los sitios tempranos han sido detectados en áreas que albergaron antiguas paleolagunas pertenecientes a Aguacatenango y Teopisca (García y Santamaría, 1982). El potencial de las paleolagunas para atraer a poblaciones humanas tempranas queda demostrado por las ocupaciones ya detectadas por García-Bárcena y colaboradores. En cuanto a las tierras bajas de la Depresión Central (ver Foto 1), la presencia de yacimientos de fósiles del pleistoceno tardío de diversos animales - tortugas (Kinosternon scorpioides, Trachemys scripta, cf. Staurotypus), mamíferos como bisontes (Bison sp.), además de mamuts y equinos (Mammuthus columbi y Equus conversidens) - indican la abundancia y disponibilidad de estos recursos faunísticos (Luna-Espinosa y Carbot-Chanona, 2009).
Una explotación de nichos ecológicos heterogéneos sería consistente con la propuesta de Acosta (2008 y 2014; Acosta, Gómez y García, 2016) sobre cazadores recolectores con tecnologías expeditivas, quienes no se especializaron en la cacería de megafauna. Aunque aún no se registra evidencia de actividades humanas en los yacimientos con fauna pleistocénica, no dejan de ser puntos que guardan un alto potencial y que deberían ser examinados con mayor detalle y sistematicidad en el futuro.

\section{Conclusiones}

En resumen, las ventajas de generar modelos como el anteriormente expuesto conducirán a un conocimiento más amplio de las áreas de aprovechamiento y tránsito de los primeros habitantes del continente americano, y a través de estas predicciones podrán mejorarse los métodos para la detección y estudio de sitios arqueológicos de sociedades nómadas y cazadoras recolectoras.

En este trabajo se presentó una propuesta para la identificación de rutas óptimas entre sitios precerámicos y con ello contribuir al debate relacionado con las diferentes rutas de poblamiento temprano en América. Al simular de forma más detallada las rutas por donde los primeros pobladores pudieron transitar, independientemente de su origen - Beringia Atlántico Norte, etcétera-, se contará con mayores oportunidades para entender sus ritmos de adaptación a los entornos con los que se topaban. Naturalmente, el presente estudio es preliminar y la búsqueda de información significativa para afinar la precisión del método expuesto continúa explorándose. De forma lógica, su pertinencia sólo podrá ser evaluada cuando se efectúen exploraciones en campo siguiendo los preceptos aquí sugeridos.

\section{Notas}

${ }^{1}$ Ver: Dillehay et al., 1992; Meltzer, 1995; Anderson y Gillam, 2000; Dixon, 2001; Fiedel, 2000; Bradley y Stanford, 2004; Lanata, 2011; Acosta, 2014; Ardelean, 2014. 
${ }^{2}$ En este trabajo se utiliza el término precerámico para abarcar a todas las sociedades que desarrollaron un modo de vida de cazador-recolector, previo al surgimiento de las primeras aldeas, la agricultura y la cerámica (ca. 5000 a.p.).

${ }^{3}$ Ver: Acosta 2008; 2010; 2011; García y Santamaría, 1982; MacNeish y Peterson, 1962; Voorhies et al., 2002.

${ }^{4}$ Ver, entre otros: MacNeish y Peterson, 1962; García y Santamaría, 1982; Acosta 2008, 2014.

${ }^{5}$ Ver: Limp, 1996; Wheatley y Gillings, 2002; Fábrega, 2004; Chapman, 2006; Grau, 2006; Jones, 2006.

${ }^{6}$ Se consultaron, por ejemplo, las siguientes fuentes: Lorenzo, 1977; García, 1982; García y Santamaría, 1982; Acosta, 2008; López-Bravo y Macías, 2016.

7 Se consultaron, entre otras, las siguientes fuentes: Carbot-Chanona, 2010; Carbot-Chanona y GómezPérez, 2014; Luna-Espinosa y Carbot-Chanona, 2009.

\section{Referencias}

Acosta Ochoa, Guillermo (2008). La cueva de Santa Marta y los cazadores-recolectores del Pleistoceno final-Holoceno temprano en las regiones tropicales de México. Tesis doctoral. Ciudad de México: Universidad Nacional Autónoma de México.

Acosta Ochoa, Guillermo (2010). "Late-Pleistocene/ Early-Holocene Tropical Foragers of Chiapas, Mexico: Recent Studies". En Current Research in the Pleistocene, 27: 1-4.

Acosta Ochoa, Guillermo (2011). "El poblamiento de las regiones tropicales de México hace 12,500 años”. En Anales de Antropología, 45: 227-235.

Acosta Ochoa, Guillermo (2014). "Nuevos estudios sobre las sociedades precerámicas de Chiapas". En Eduardo Corona Martínez y Joaquín Arroyo Cabrales (coords.), Perspectivas de los estudios de prehistoria en México. Un homenaje a la trayectoria del ingeniero Joaquín García-Bárcena. México: INAH, pp. 143-168.

Acosta Ochoa, Guillermo, Luis Fernando Gómez Padilla y Víctor Hugo García Gómez (2016). “Modelos SIG como herramienta heurística en el análisis del poblamiento de México y Centroamérica”. En J.C. Jiménez López, Carlos Serrano Sánchez, Berna Valles Canales, Felisa
Aguilar Arellano, Arturo González González y Carlos E. López (eds.), El poblamiento temprano en América 6. Saltillo, Coahuila: Museo del Desierto, pp. 109-127.

Anderson, David G. y J. Christopher Gillam (2000). "Paleoindian Colonization of the Americas: Implications from an Examination of Physiography, Demography, and Artifact Distribution". En American Antiquity, 65(1): 43-66.

Ardelean, Ciprian (2014). "The Early Prehistory of the Americas and the Human Peopling of the Western Hemisphere. An Overview of Archaeological Data, Hypotheses and Models". En Studii de Preistorie, 1l: 33-95.

Bettinger, Robert L. (1991). Hunter-Gatherers. Archaeological and Evolutionary Theory. Nueva York y Londres: Plenum Press.

Bradley, Bruce y Denis Stanford (2004). "The North Atlantic Ice-Edge Corridor: a Possible Palaeolithic Route to the New World". En World Archaeology, 36: 459-478.

Butzer, Carl (1989). Arqueología, una ecología del hombre: método y teoría para un enfoque contextual. Barcelona: Bellaterra.

Carbot-Chanona, Gerardo (2010). "The First Record of Dasypus (Xenarthra: Cingulata: Dasypodidae) in the Late Pleistocene of México". En Current Research in the Pleistocene, 27: 164-166.

Carbot-Chanona, Gerardo y L.E. Gómez-Pérez (2014). "Nueva evidencia de Panthera atrox (Mammalia, Felidae) en el Pleistoceno Tardío de Chiapas". En Lacandonia, 8: 83-89.

Chapman, Henry (2006). Landscape archaeology and GIS Gran Bretaña: Tempus Pub Limited.

Dillehay, Thomas D, Gerardo Ardila Calderón, Gustavo Politis y Maria da Conceicao de Moraes Coutinho Beltrao (1992). "Earliest Hunters and Gatherers of South America". En Journal of World Prehistory, 6(2): 145-204.

Dixon, E. James (1999). Bones, Boats, and Bison: Archaeology and the First Colonization of Western North America. Albuquerque: University of New Mexico Press.

Dixon, E. James (2001). "Human Colonization of the Americas: Timing, Technology and Process". En Quaternary Science Reviews, 20: 277-299. 
Fábrega Álvarez, Pastor (2004). "Poblamiento y territorio de la cultura castreña en la comarca de Ortegal". En Cuadernos de Arqueoloxía e Patrimonio, Laboratorio de Patrimonio, Paleoambiente e Paisaxe, 19. España: IIT-USC.

Fiedel, Stuart (1996). Prehistoria de América. Barcelona: Crítica.

Fiedel, Stuart (2000). "The Peopling of the New World: Present Evidence, New Theories, and Future Directions". En Journal of Archaeological Research, 8(1): 39-103.

Fladmark, Knut R. (1979). "Routes: Alternate Migration Corridors for Early Man in North America". En American Antiquity, 44: 55-69.

Fladmark, Knut R. (1986). “The First Americans. Getting One’s Berings”. En Natural History, 95: 8-19.

Flannery, Kent (ed.) (1986). Guila Naquitz. Archaic Foraging and Early Agriculture in Oaxaca, Mexico. Cambridge: Academic Press.

García Bárcena, Joaquín (1982). El precerámico de Aguacatenango, Chiapas, México. México: INAH.

García Bárcena, Joaquín y Diana Santamaría (1982). La cueva de Santa Marta Ocozocoautla, Chiapas, Estratigrafía, cronología cerámica. Tuxtla Gutiérrez: INAH.

Grau Mira, Ignacio (2006). La aplicación de los SIG en la arqueología del paisaje. Alicante: Universidad de Alicante.

Jackson Jr., L.E., F.M. Phillips, K. Shimamura y E.C. Little (1996). "Quaterary Geology of the Ice-free Corridor: Glacial Controls on the peopling of the new world". En Takeru Akazawa y Emoke Szathmáry (eds.), Prehistoric Mongoloid Dispersals. Nueva York: Oxford University Press, pp. 214-227.

Johnson, Eilen, Joaquín Arroyo-Cabrales y Oscar J. Polaco (2006). "Climate, Environment, and Game Animal Resources of the Late Pleistocene Mexican Grassland". En José Concepción Jiménez López, Silvia González, José Antonio Pompa y Padilla y Francisco Ortiz Pedraza (coords.), El hombre temprano en América y sus implicaciones en el poblamiento de la cuenca de México. Primer simposio Internacional. México: INAH, pp. 231-246.

Jones, Erick (2006). "Using Viewshed Analysis to Explore Settlement Choice: A Case Study of the Onondaga Iroquois". En American Antiquity, 71: 523-538.
Josenhans, H., Fedje, D., Pienitz, R. y Southon, J. (1997). "Early Humans and Rapidly Changing Holocene Sea Levels in the Queen Charlotte Islands-Hecate Strait, British Columbia, Canada”. En Science, 277: 71-74.

Lanata, José Luis (2011). "Discutiendo diferentes modelos de la dispersión humana en las Américas". En José Concepción Jiménez, Carlos Serrano, Arturo González y Felisa J. Aguilar Arellano (eds.), IV Simposio Internacional. El hombre temprano en América. México: INAH, UNAM, Museo del Desierto, pp. 121-143.

Lee, Thomas (1985). "El asentamiento humano precolombino del valle de Hueyzacatlan". En San Cristóbal y sus alrededores. Tuxtla Gutiérrez: Gobierno del Estado de Chiapas, pp. 153-186.

Limp, W. Frederick (1996). "Developing Methodologies in the Analysis of Spatially Referenced Data and their Impacts on Archaeological Method and Theory". En A.C. Amilcare Bietti, A. Cazzella, Ian Johnson y Albertus Voorrips (eds.), The Colloquia of the XIII International Congress of Prehistoric and Protohistoric Sciences, 1: Theoretical and Methodological Problems. Forli, Italia: Abaco, pp. 115-126.

López-Bravo, Roberto y Juan Ignacio Macías Quintero (2016). Informe técnico parcial de la primera temporada del proyecto "Economía y sociedad en el periodo Postclásico: comunidad y unidades domésticas en Chiapa de Corzo". Tuxtla Gutiérrez: Facultad de Humanidades, Escuela de Arqueología, UNICACH.

Lorenzo, José Luis (1977). Un conjunto lítico de Teopisca, Chiapas. México: Instituto Nacional de Antropología e Historia.

Luna-Espinosa, Josué R. y Gerardo Carbot-Chanona (2009). "First Record of Late-Pleistocene Turtles from Chiapas, Mexico". En Current Research in the Pleistocene, 26: 162-164.

Macías Quintero, Juan Ignacio (2017). Los cazadores recolectores del semidesierto de Zacatecas, México. Un estudio arqueológico. Tesis de doctorado. México: IIA-UNAM. MacNeish, Richard y Peterson Frederick (1962). The Santa Marta Rock Shelter Ocozocoautla, Chiapas, Mexico. Utah: Brigham Young University.

Meltzer, Daniel. J. (1995). "Clocking the First Americans". En Annual Review of Anthropology, 24: 21-45. 
Pérez-Crespo, Víctor Adrían, Gerardo Carbot-Chanona, Pedro Morales-Puente, Edith Cienfuegos-Alvarado y Francisco J. Otero (2015). "Paleoambiente de la Depresión Central de Chiapas, con base en isótopos estables de carbono y oxígeno". En Revista Mexicana de Ciencias Geológicas, 32: 273-282.

Pincemin, Sophia (1990). "INAH y la prehistoria de Chiapas". En Primer Foro de Arqueología de Chiapas: cazadores-recolectores-pescadores, agricultores tempranos. Tuxtla Gutiérrez: Gobierno del Estado de Chiapas, Instituto Chiapaneco de Cultura, pp. 21-27.

Santamaría Estévez, Diana (2014), "Los sitios precerámicos de Chiapas: 1974-1984”. En Eduardo Corona Martínez y Joaquín Arroyo Cabrales (coords.), Perspectivas de los estudios de prehistoria en México. Un homenaje a la trayectoria del ingeniero Joaquín García-Bárcena. México: INAH, pp. 49-62.

Stanford, Dennis y Bruce Bradley (2002). "Ocean Trails and Prairie Paths? Thoughts about Clovis Origins". En Nina Jablonsky (ed.), The First Americans: the Pleistocene Colonization of the New World. San Francisco: California Academy of Sciences, pp. 255-271.

Stemp, W. James, Jaime J. Awe, Keith M. Prufer y Christopher G.B. Helmke (2016). "Design and Function of Lowe and Sawmill Points from the Preceramic Period of Belice". En Latin American Antiquity, 27(3): 279-299.
Trujillo, Armando (2011). Un modelo predictivo. El caso de la minería del cobre durante el siglo XVI en la región de tierra caliente, Michoacán. Zamora, Michoacán: El Colegio de Michoacán.

Voorhies, Barbara, D.J. Kennett, J.G. Jones y T.A. Wake (2002). "A Middle Archaic Archaeological Site on the West Coast of Mexico". En Latin American Antiquity, 13: 179-200.

Warren, R.E. y D.L. Asch (2000). “A Predictive Model of Archaeological Site Location in the Eastern Prairie Peninsula". En Konnie L. Wescott y R. Joe Brandon (ed.), Practical Applications of GIS for Archaeologists: a Predictive Modelling Toolkit. Londres: Taylor \& Francis.

Wheatley, David y M. Gillings (2002). The Archaeological Applications of GIS. Londres y Nueva York: CRC Press. Winter, Marcus (2014). "La prehistoria en Oaxaca: avances recientes". En Eduardo Corona Martínez y Joaquín Arroyo Cabrales (coords.), Perspectivas de los estudios de prehistoria en México. Un homenaje a la trayectoria del ingeniero Joaquín García-Bárcena. México: INAH, pp. 123-142.

Winterhalder, Bruce (2003). "The Behavorial Ecology of Hunter-Gatherers". En Catherine Panter-Brick, Robert H. Layton y Peter Rowley-Conwy (eds.), HunterGatherers: An Interdisciplinary Perspective. Cambridge, Reino Unido: Cambridge University Press, pp. 12-38. 
Figura 1. Procedimiento de trabajo empleado en la investigación



Fuente: elaboración propia.

Foto 1. Paisaje actual de la Depresión Central de Chiapas en el verano de 2017

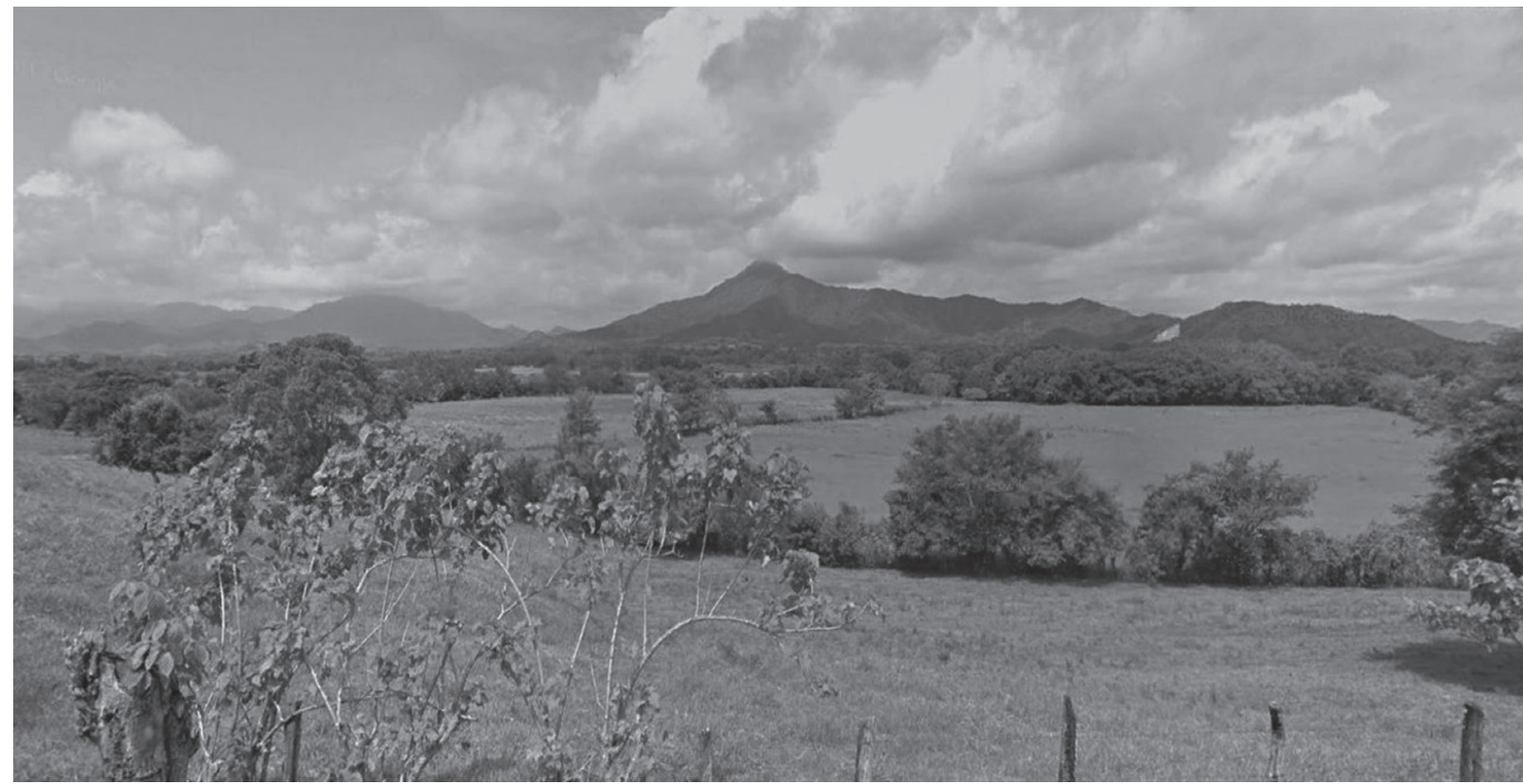

Fuente: fotografía de Stephanie Reyes Ibelles. 
Mapa 1. Rutas hipotéticas de colonización temprana en América

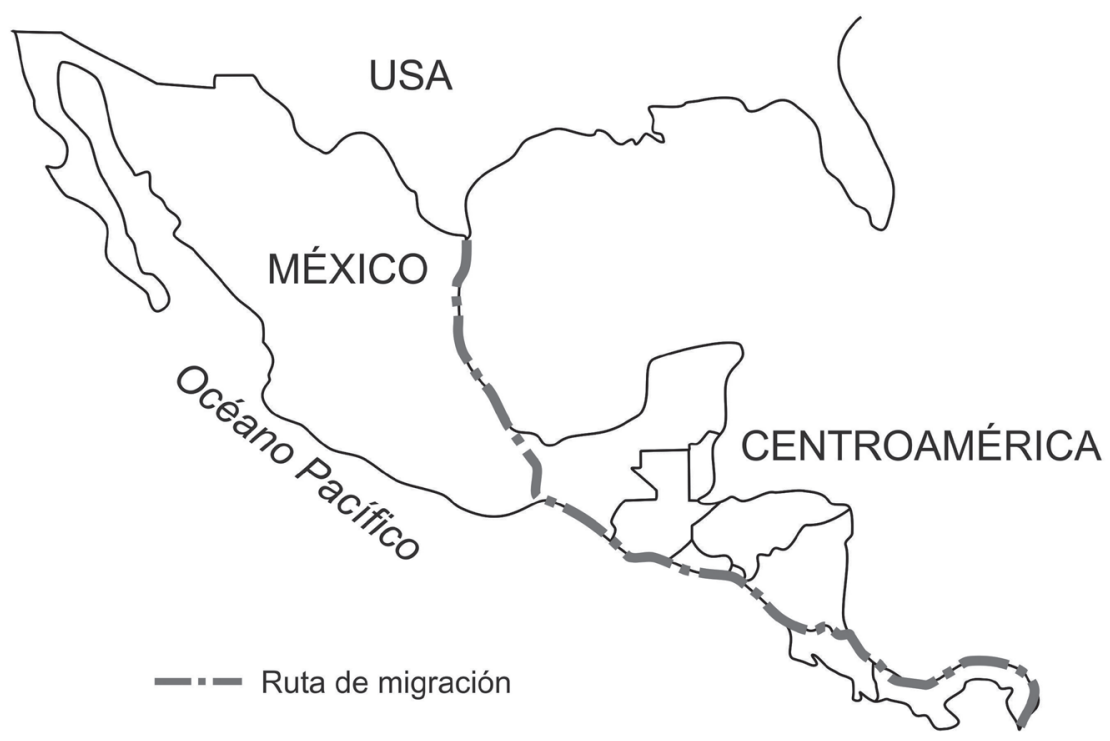

Fuente: elaboración propia, sobre mapa de Anderson y Gillman, 2000: 50.

Mapa 2. Ubicación del área de estudio y localidades mencionadas en el texto

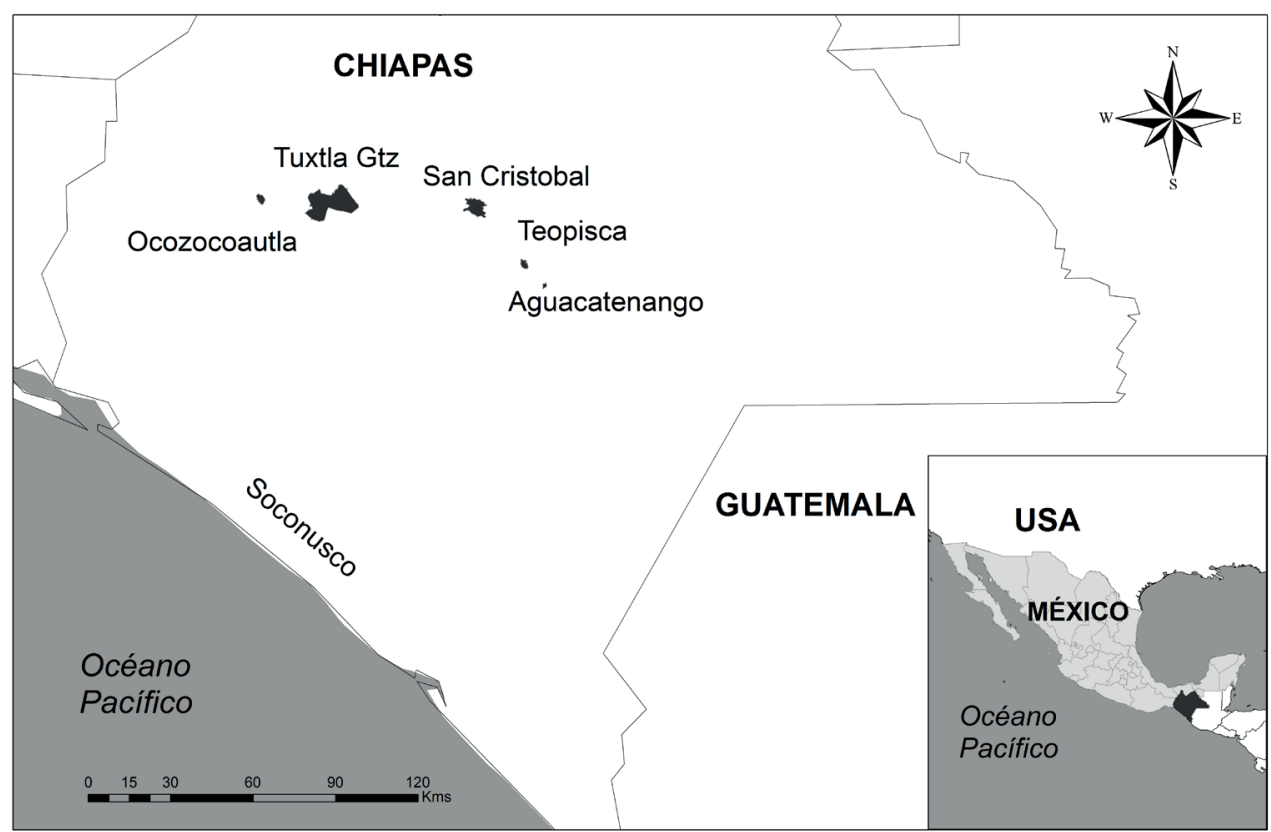

Fuente: elaboración propia. 
Mapa 3. Uso de Global Mapper para obtener curvas de nivel del área de estudio

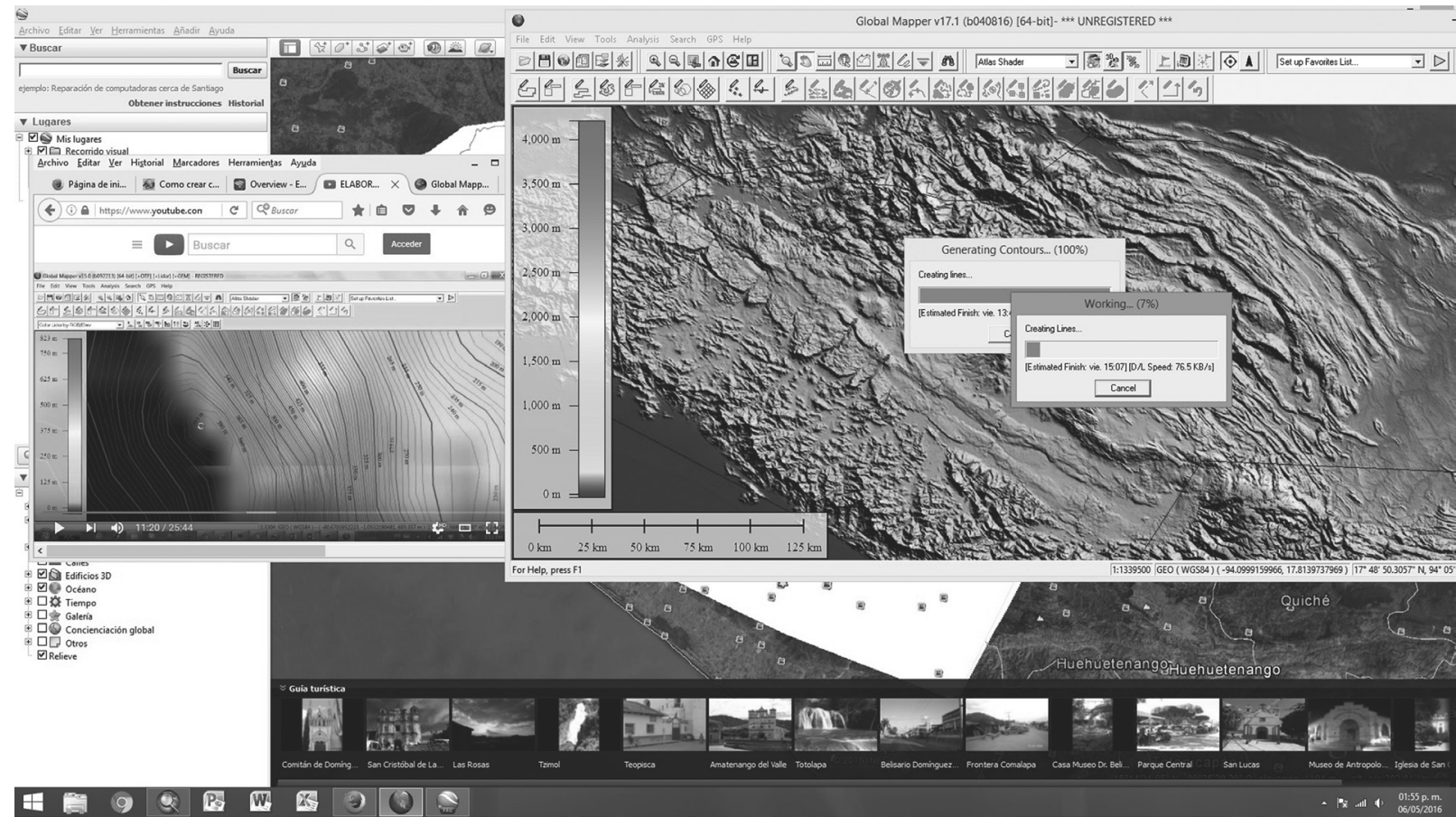

Fuente: elaboración propia.

Mapa 4. Proceso de caracterización del DEM y los sitios analizados. Modelo de pendientes

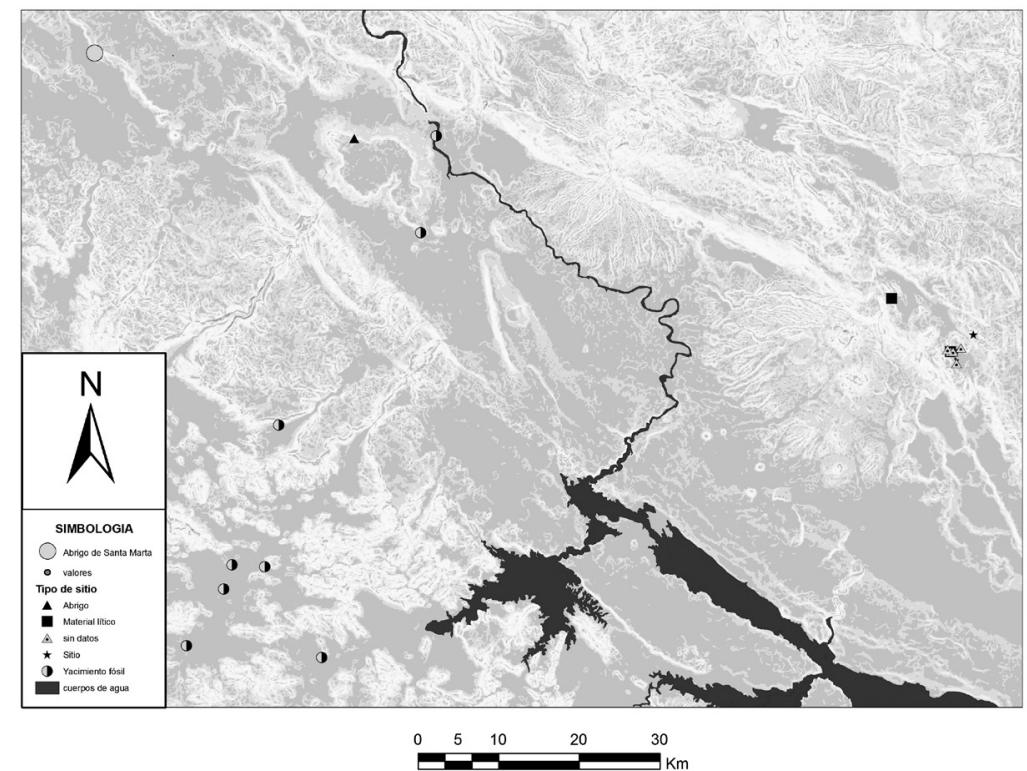

Fuente: elaboración propia. 
Mapa 5. Proceso de caracterización del DEM y los sitios analizados. DEM que muestra la dirección de las pendientes


Fuente: elaboración propia. 
Mapa 6. Proceso de caracterización del DEM y los sitios analizados.

Muestra la relación costo-distancia a partir del sitio de Santa Marta

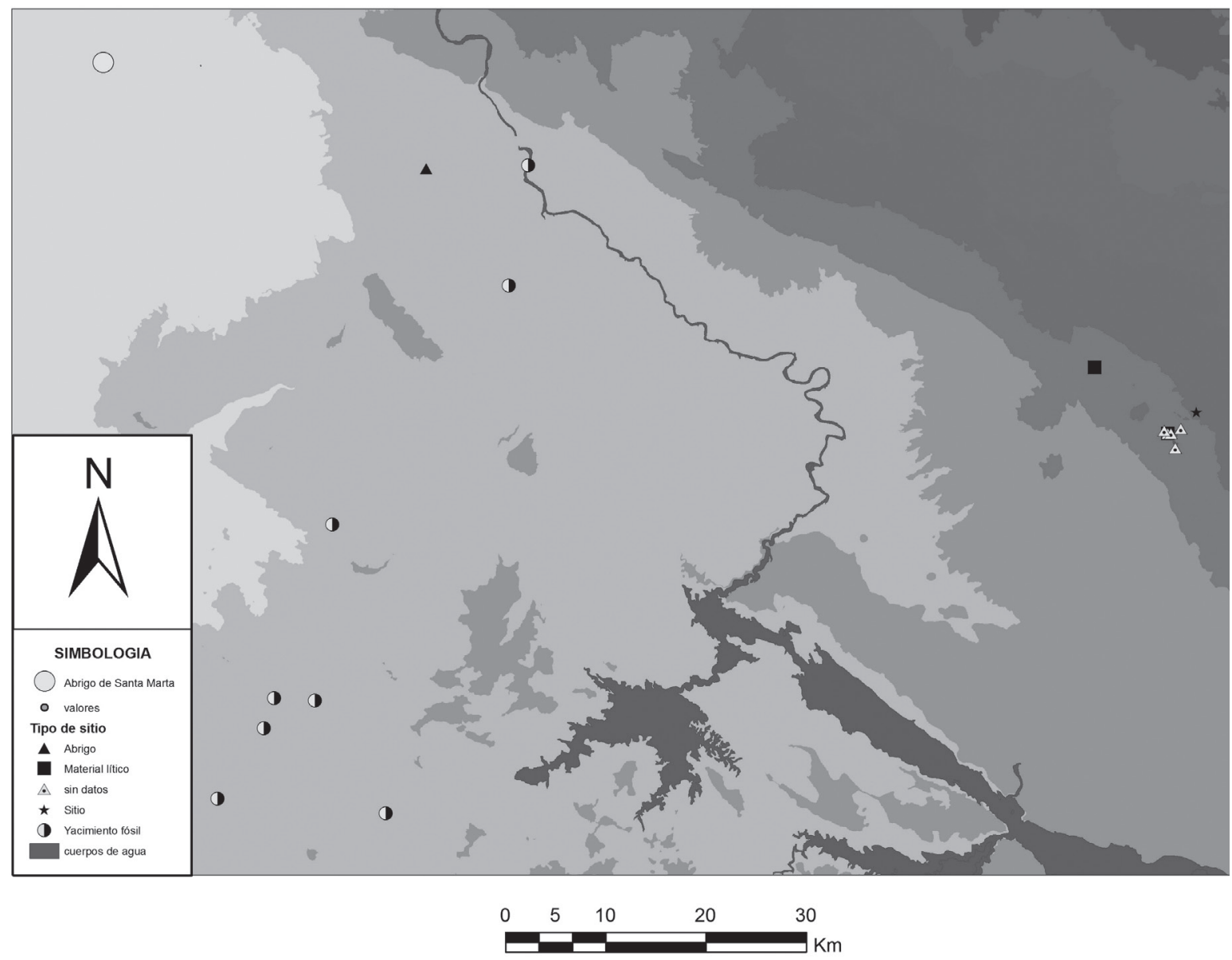

Fuente: elaboración propia. 
Mapa 7. Muestra las rutas óptimas de menor costo que comunicarían al abrigo de Santa Marta con otros sitios precerámicos
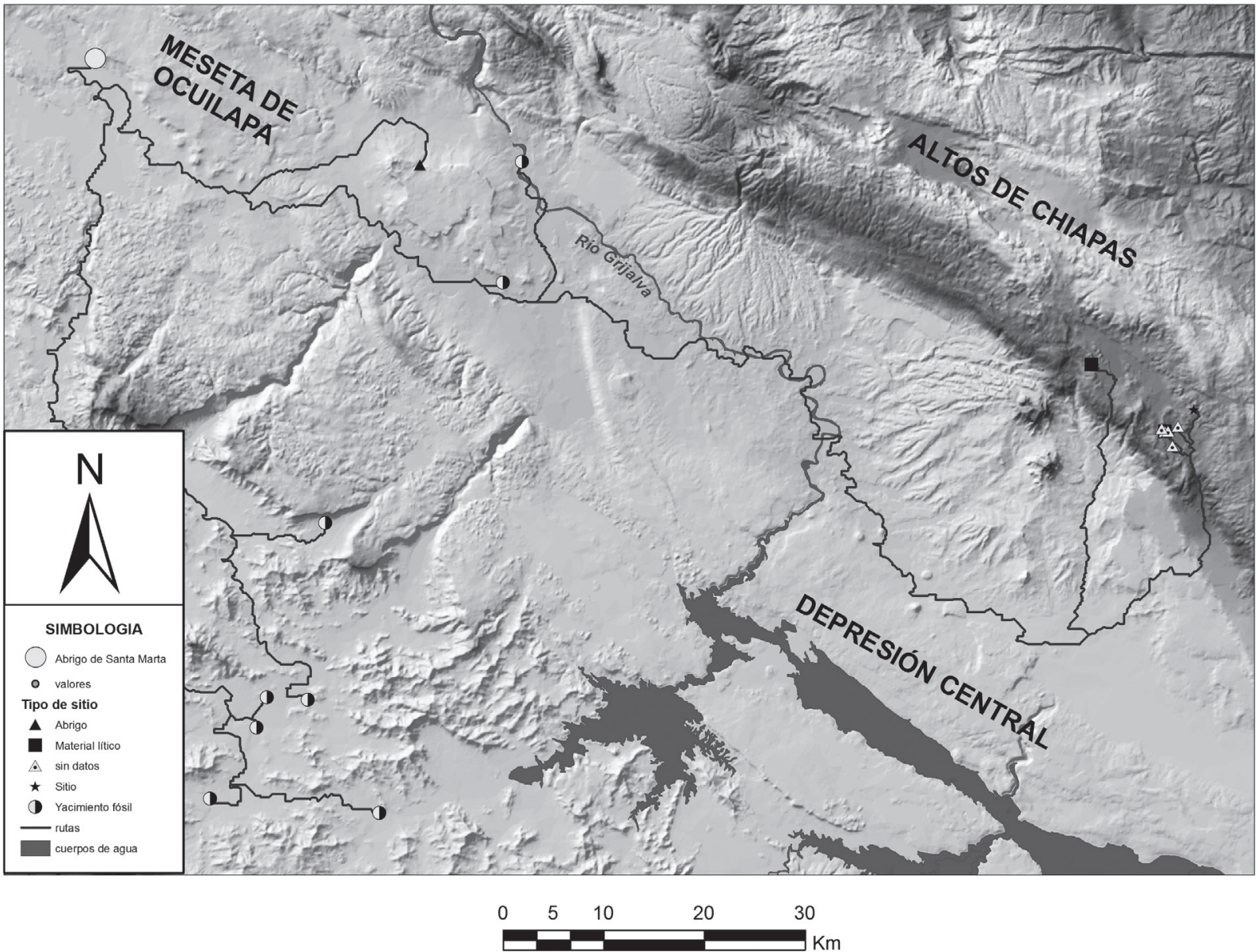

Fuente: elaboración propia 